\title{
ПРИНЦИПЫ ФОРМИРОВАНИЯ СИСТЕМЫ СТРАТЕГИЧЕКОГО УПРАВЛЕНИЯ ПРОЦЕССАМИ ЦИФРОВИЗАЦИИ В МУНИЦИПАЛЬНЫХ ОБРАЗОВАНИЯХ
}

\author{
Лившиц В.Н., Мызникова М.Н., Дмитриева О.В.
}

Российские муниципальные образования играют ключевую роль в национальной экономике, однако все чаще сталкиваются с вызовами, которые препятствуют их полноченному развитию. Основной целью данной статьи является определение основных концептуальных принципов формирования системы стратегического управления на уровне муниципальных образований, что будет способствовать повышению качества жизни населения.

DOI: $10.20537 /$ mce2021econ03

Социально-экономический и демографический вес городов в современном мире ставит принципиально новые задачи для формирования эффективной стратегии развития города. Рост миграции, избыточная плотность, транспортные проблемы, экологическое давление, изменение требований жителей и бизнеса к качеству городской среды и предоставляемых услуг являются вызовами к поиску механизмов их решения.

В этих условиях происходит постепенный пересмотр подходов к управлению городом, все более очевидным становится необходимость в использовании передовых технологических решений.

Одним из приоритетных шагов в экономическом развитии многих стран стал переход к цифровой экономике. Важность развития цифровой экономики на сегодняшний день осознают не только традиционные экономические лидеры (США, Германия, Великобритания, Япония и др.), но и многие развивающиеся страны. Не является исключением в этом списке и Россия.

Цифровизация проявляется прежде всего в развитии робототехники, технологий беспроводной связи, интернета вещей, искусственного интеллекта. Внедрение подобных технологий способно повысить производительность труда в компаниях до 40\%. Для России проблемы повы- 
шения производительности труда практически во всех отраслях экономики являются одними из наиболее актуальных и переход к цифровой экономике может позволить ускоренными темпами сократить разрыв в производительности труда с рядом зарубежных стран, позиции которых в этой области значительно превосходят российские.

Внедрение и эффективное использование цифровых технологий во многом будет определять международную конкурентоспособность как отдельных компаний, так и страны в целом. В этой связи стремление России уйти от сырьевой зависимости и повысить свою роль в мировой экономике может быть реализовано лишь при повышении внутренней и внешней конкурентоспособности компаний во многом благодаря цифровизации.

В переходе к цифровой экономике большинство стран видят массу преимуществ, однако стоит выделить и негативные последствия, которые несет собой цифровизация. Прежде всего, это замена многих профессий автоматизированными системами и программами, исчезновение традиционных сфер деятельности, угрозы утери конфиденциальной информации и ущемление прав человека в цифровом пространстве, неминуемое увеличение киберпреступности. Однако преимуществ от цифровизации страна и компании могут получить значительно больше, чем угроз, а сокращению последних будет способствовать совершенствование правовых норм в новых условиях цифровой экономики.

В целом, цифровизация российской экономики должна стать одним из важнейших источников долгосрочного экономического роста. По оценке экспертной группы Digital McKinsey, потенциальный эффект для ВВП от цифровизации экономики к 2025 г. за десять лет может составить от 4.1 до 8.9 трлн руб., что обеспечит от 19 до 34\% общего увеличения ВВП за этот период.

Один из ожидаемых результатов цифровизации экономики - повышение качества жизни граждан, которое должно проявиться в следующем:

- рост покупательской способности населения;

- новые возможности трудоустройства, привлекательность экономики для квалифицированных кадров;

- комфортные для жизни города;

- доступная и качественная медицина и образование;

- удобные цифровые сервисы и госуслуги.

Цифровая трансформация экономики страны предопределяет изменения в стратегическом управлении на муниципальном уровне. При этом 
Лившии В.Н., Мызникова М.Н., Дмитриева О.В. Принципь формирования.., стр. 37-48 Livchits V.N., Myznikova M.N., Dmitrieva O.V. Principles of the formation..., pp. 37-48

система стратегического управления процессами цифровизации в муниципальных образованиях должна строиться на определенных принципах.

Принцип многоуровневости. Принцип многоуровневости предполагает наличие множества уровней в системе управления процессами. Он позволяет исследовать:

- иерархии связей структурных компонентов системы;

- высший, средний и низший уровень управления системой;

- общие, особенные и единичные свойства системы;

- подсистемы, части и элементы системы в ее структуре.

Многоуровневость в социально-экономической системе означает, что в ней существует огромное количество связей между различными элементами, образующими тот или иной уровень организации, то есть это сложная иерархически взаимосвязанная структура.

С позиции масштабов управления в национальной экономике выделяются следующие три уровня:

- макросистемы - крупнейшие территориальные системы, например, территория страны или какой-либо автономии внутри государства;

- мезосистемы - территории средней величины, например, в РФ это территории федеральных округов или субъектов РФ;

- микросистемы - малые территориальные социально-экономические системы, например, город и муниципальные образования.

Принцип многоуровневости для муниципальных образований, относящихся к микросистеме, означает встроенность в общую систему уровней управления в экономике, взаимосвязь и взаимозависимость между этими уровнями.

Формирование системы стратегического управления процессами цифровизации в муниципальных образованиях во многом зависит от глубины проникновения цифровых решений и сервисов в субъекте РФ, на территории которого располагается муниципальное образование. При этом цифровая развитость самого субъекта РФ зависит не только от расторопности его руководства в части реализации цифровых технологий, но и финансовых возможностей региона.

Система стратегического управления процессами цифровизации в муниципальных образованиях не может не учитывать эколого-экономический фактор развития территории. И здесь так же можно выделить принцип многоуровневости в управлении устойчивым развитием. 
Эколого-экономическое благополучие обеспечивается на следующих уровнях [1]:

- межстрановой уровень, основанный на эколого-экономической политике, направленной на стимулирование экологизации производственной сферы и получение преимуществ на международной арене за счет использования механизмов гибкости, предусмотренных Киотским протоколом и другими международными соглашениями;

- национальный уровень, предполагающий трехуровневую систему моделей «регион - отрасль - предприятие», обеспечивающую оптимизацию процесса выделения средств на экологические и социальные нужды регионов и модернизацию инфраструктуры, обеспечивающих на отраслевом уровне эффективное развитие российских регионов при оптимальном объеме выпускаемой продукции;

- региональный уровень с формированием программ экологической направленности в рамках территориальных и отраслевых программ социально-экологического развития территорий субъекта РФ с активизацией внедрения цифровых технологий и инноваций в экологически чистое производство и снижение негативного воздействия от деятельности «экологически грязных» отраслей;

- производственный уровень, предполагающий процесс экологизации производственной деятельности с использованием перспективных подходов и приемов повышения эффективности использования ресурсов и совершенствования эколого-экономической политики на различных уровнях управления производственно-хозяйственными и иными комплексами.

Муниципальные образования выступают в данном случае составной частью регионального уровня в рамках рассматриваемого принципа. Во многом муниципальные образования могут быть важным связующим звеном между региональным и производственным уровнями, поскольку экологизация прежде всего направлена на обеспечение комфортной среды для граждан, проживающих в городах и муниципальных образованиях, где муниципалитеты осуществляют управление процессами.

Принципь системного анализа. В системном анализе любой объект рассматривается с учетом его системного характера, то есть не как единое целое, а как комплекс взаимосвязанных составных элементов, их свойств и процессов.

Наиболее широкое распространение системный анализ получил в теории и практике управления - при поиске возможностей, принятии и обосновании решений, связанных с проектированием, созданием и 
управлением сложными, многоуровневыми и многокомпонентными (социальными, экономическими, организационными и др.) системами.

Системный анализ может быть полезен при выработке и рассмотрении альтернативных подходов к проблемам образования, здравоохранения, городского транспорта, природных ресурсов, загрязнения окружающей среды и многочисленных других проблем [2, с. 72]. Многие из этих проблем относятся к ведению муниципалитетов, поэтому при формировании системы стратегического управления процессами цифровизации в муниципальных образованиях системный анализ может играть весьма заметную роль.

Теоретическую и методологическую основу системного анализа составляет системный подход, а также методы исследований с использованием подходящих теорий моделирования, математической логики, и статистики.

Системный подход - направление методологии специального научного познания и социально-экономической практики, в основе которого лежит исследование объектов как систем, опирающееся на использование принципа системности [2, с. 63].

Основу построения методики анализа и синтеза систем в конкретных условиях составляет перечень принципов системного анализа, которые представляют собой обобщение практики работы со сложными системами. Наиболее часто к системным причисляют следующие принципы.

Принции конечной цุели. Этот принцип подразумевает приоритет конечной (глобальной) цели, достижению которой должна быть подчинена деятельность системы [3].

Применительно к муниципальному образованию цель определяется как состояние, которое необходимо достичь к определенному моменту времени, затратив на это определенные ресурсы (материальные, человеческие и другие, имеющие ограниченный характер).

Принции измерения. О качестве функционирования какой-либо системы можно судить только применительно к системе более высокого порядка. Это значит, что для определения эффективности функционирования системы следует представить ее как часть более общей и проводить оценку внешних свойств исследуемой системы относительно целей и задач [3].

Муниципалитеты относятся к местным органам власти, которые функционируют в общей системе с федеральными и региональными структурами управления. Измерение эффективности деятельности муниципального образования не может не учитывать процессы, происходящие на федеральном и региональном уровне: экономические реформы, 
инвестиционная политика, социальная политика, совершенствование нормативно-правовой базы и др.

Принцип единства. В соответствии с этим принципом систему следует рассматривать как целое, состоящее из отдельных, связанных между собой определенными отношениями, частей (элементов) [3].

Применительно к муниципальным образованиям, они являются составной частью системы управления процессами в стране.

Принщип связности. Рассмотрение любой части совместно с ее окружением подразумевает проведение процедуры выявления связей между элементами рассматриваемой системы и выявление связей с внешней средой (учет внешней среды) [3].

В соответствии с этим принципом систему управления на муниципальном уровне следует рассматривать как часть (подсистему) другой системы более высокого уровня, называемой старшей системой.

Принции иерархии. В соответствии с этим принципом осуществляется введение иерархии частей рассматриваемой системы и их ранжирование, что упрощает разработку системы и устанавливает порядок рассмотрения частей [3]. Иерархия свойственна всем сложным системам.

Иерархия в структурах организационных систем (включая систему муниципальных образований) связана с характером управления в системе, степенью децентрализации управления.

Принции цุентрализащчии и децентрализащчии. Этот принцип подразумевает сочетание в сложных системах централизованного и децентрализованного управления, которое, как правило, заключается в том, что степень централизации должна быть оптимальной, обеспечивающей наиболее эффективное выполнение поставленной цели [3]. Вместе с тем, основной традиционный недостаток децентрализованного управления увеличение времени адаптации системы, сложность управления, связанная со значительными объемами потоков информации, подлежащей переработке в старшей системе управления.

Для решения данной проблемы используется внедрение цифровых технологий, связанных с обработкой «больших данных», что позволяет субъектам экономики на местном уровне максимально быстро, своевременно и качественно реагировать на изменяемые условия, отвечать потребностям граждан, создавать лучшие условия для работы бизнес-структур.

Принции функциональности. В соответствии с этим принципом структура и функции в исследуемой системе рассматриваются совместно и с приоритетом функции над структурой. Данный принцип утверждает, 
что любая структура тесно связана с функцией системы и ее составных частей. В случае придания системе новых функций, как правило, пересматривается и ее структура. Поскольку выполняемые функции составляют процессы, то целесообразно рассматривать отдельно процессы, функции, структуры.

В организационных системах структура создается после определения набора функций и реализуется в виде совокупности персонала, методов, алгоритмов, технических устройств различного назначения. При появлении новых задач и соответственно функций может оказаться необходимой корректировка структуры [3].

Система управления на муниципальном уровне как составная часть общей системы управления с целью повышения эффективности работы должна предполагать изменение и возможно перераспределение функций внутри общей системы, что оказывает влияние на корректировку структуры управления на рассматриваемом уровне.

Принции развития. Этот принцип подразумевает учет изменяемости системы, ее способности к развитию, адаптации, расширению, замене частей, накапливанию информации. В основу синтезируемой системы требуется закладывать возможность развития, наращивания, усовершенствования [3].

Цифровые технологии, внедряемые на разных уровнях управления, позволяют расширять возможности для социально-экономического развития субъектов РФ, входящих в них муниципальных образований.

Вместе с тем учесть все факторы изменяемости системы как правило невозможно. В этом случае можно выделить принцип неопределенности. Этот принцип подразумевает учет неопределенностей и случайностей в системе и является одним из основных принципов системного подхода. В соответствии с этим принципом считается, что можно иметь дело с системой, в которой структура, функционирование или внешние воздействия не полностью определены [3].

Как правило, сложные открытые системы не подчиняются вероятностным законам. При анализе таких систем могут быть получены зачастую лишь вероятностные оценки прогнозируемых ситуаций, если эти оценки объективно существуют. Однако такие прогнозные оценки должны включать как минимум три варианта развития ситуации: оптимистический, пессимистический (шоковый, стрессовый) и базовый, в зависимости от влияющих на событие факторов и степени влияния каждого из них. 
Принципь Эшби. Проблема нахождения оптимальной модели управления состоит в сложности воздействий, оказываемых окружающей средой на объект управления. Критерием оценки сложности может служить разнообразие, то есть количество возможных состояний, которые может принимать система.

У.Р. Эшби сформулировал закон необходимого разнообразия: «Чтобы управление системой было возможно, разнообразие управляющих действий должно быть не меньше разнообразия возмущений на входе в систему» [4].

По определению Эшби, данный закон заключается в том, что разнообразие сложной системы требует управления, которое само обладает некоторым разнообразием.

Область действия этого закона распространяется на различные системы, в том числе и на управление коллективами людей и процессами человеческой деятельности. Исходя из этого рассмотрение данного закона вполне логично в деятельности муниципалитетов, в том числе в рамках системы стратегического управления процессами цифровизации в муниципальных образованиях.

Из этого закона непосредственно следует, что желательно иметь разнообразие возможных действий управляющего не ниже разнообразия вероятных действий управляемой им системы. Только в этом случае возможен надежный контроль исходов возникающих ситуаций. Или можно сказать так: разнообразие сложной системы требует управления, которое само обладает некоторым разнообразием. Иначе говоря, значительное разнообразие воздействующих на большую и сложную систему возмущений требует адекватного им разнообразия ее возможных состояний [5, с. 136].

Процесс управления, в конечном счете, сводится к уменьшению разнообразия состояний управляемой системы, к уменьшению ее неопределенности. В соответствии с этим законом, с увеличением сложности управляемой системы сложность управляемого блока также должна повышаться.

В современных условиях почти неизбежное усложнение аппарата управления должно приводить не к увеличению объема трудовых ресурсов, а к внедрению цифровых прогрессивных технологий управления, в том числе и на муниципальном уровне.

Закон необходимого разнообразия имеет принципиальное значение для разработки оптимальной структуры системы управления. Если центральный орган управления при сохранении разумных размеров не обладает 
необходимым разнообразием, то следует развивать иерархическую структуру, передавая принятие определенных решений на нижние уровни и не допуская, чтобы они превращались в передаточные инстанции [5, с. 137].

В России доля принимаемых решений на муниципальном уровне низка по сравнению с региональным и федеральным уровнем в системе управления социально-экономическими процессами. При этом в ряде зарубежных (прежде всего промышленно развитых) стран спектр принимаемых решений муниципалитетами гораздо шире, чем в России и эффективность их работы гражданами воспринимается выше. Это достигается во многом благодаря широким полномочиям муниципалитетов и их близости к рядовым гражданам по сравнению с органами государственной власти высшего порядка.

Исследуя сложные системы, обратимся ко второму принципу Эшби (как развитие закона необходимого разнообразия) - принцииуу эмерджентности. Данный принцип гласит: «Чем больше система и чем больше различия в размерах между частью и целым, тем выше вероятность того, что свойства целого могут сильно отличаться от свойств частей» [4]. Чем сильнее диверсифицирована работа, чем разнообразнее задачи, стоящие перед подразделениями и отделами, тем больше отличий в локальных целях, их слабая связь с глобальными целями организации.

Несмотря на то что этот принцип прежде всего актуален для крупных корпораций и холдингов, однако его можно рассматривать и применительно к муниципалитетам, являющимся составной частью большой системы органов управления.

Россия является федеративным государством, огромной по территории страной, состоящей из 85 субъектов РФ и более 20 тысяч муниципальных образований [6]. Учитывая этот факт, принцип эмерджентности Эшби можно транслировать применительно к современным российским реалиям: «Наличие большой системы с большими различиями в размерах между составными ее частями, приводят к различиям целей этих составных частей и имеют слабую связь с глобальными целями государства на федеральном уровне». Этот вывод подтверждает тезис о необходимости децентрализации и наделения большими полномочиями субъектов РФ и муниципальные образования, прежде всего через перераспределение налоговых поступлений по бюджетам разных уровней. Подобная мера позволила бы муниципалитетам активнее внедрять современные цифровые технологии и выстраивать систему стратегического управления на своем уровне с большей эффективностью. 
Принцип Ле Шателье. Еще один принцип, рассматриваемый при формировании системы стратегического управления процессами цифровизации в муниципальных образованиях - принцип Ле Шателье-Брауна, называемый также принципом смещения равновесия, используемый в различных науках, в том числе и экономике.

Суть принципа Ле Шателье-Брауна в следующем: при внешнем воздействии, выводящем систему из состояния устойчивого равновесия, это равновесие смещается в том направлении, при котором эффект внешнего воздействия ослабляется [7].

Несколько иными словами принцип выделен Лившицем В.Н. [2, с. 63]: «В экономике внешнее воздействие, выводящее систему из состояния устойчивого равновесия, стимулирует в ней процессы, стремящиеся ослабить результат этого воздействия».

Данный принцип применяется в рамках фазового перехода от экстенсивной к интенсивной модели развития. Для сложившейся (экстенсивной) экономической системы переход на интенсивную модель является нарушающим воздействием, и она, согласно этому принципу, объективно ему противится. Тем самым недостаточно будет подготовить и запустить механизмы интенсификации, нужно еще затратить дополнительные силы и энергию на перестройку старой системы [7, с. 57].

Иными словами, для успешной реализации серьезного системного преобразования необходимо планировать дополнительный излишек средств (материальных и нематериальных ресурсов) на мероприятия по демонтажу старой системы.

Несмотря на то, что экстенсивная модель развития в современном мире давно устарела и экономическое развитие осуществляется по интенсивной модели, вместе с тем принцип Ле Шателье-Брауна можно использовать при переходе от ставшей традиционной индустриальной и постиндустриальной модели развития к цифровой экономической системе (так называемая цифровая трансформация экономики).

Данный принцип в таком случае можно применить к процессам цифровизации в муниципальных образованиях и к формированию системы стратегического управления этими процессами. Для сложившейся экономической системы (включающей уровни от федерального до местного) переход на цифровую модель развития является нарушающим воздействием, что приводит к ослаблению результата этого воздействия и тормозит трансформацию. Для получения желаемого результата требу- 
ется больше усилий, средств и времени. В условиях ограниченности ресурсов (тем более в период экономических кризисов, как например в 2020-2021 гг.) переход к цифровой экономике заметно затягивается. Однако если учесть, что большинство качественных скачков в развитии цивилизации предварялись войнами и кризисами, то управление в кризис может оказаться наиболее эффективным, т.к. имеющиеся связи предельно ослаблены и нет нужды тратить на это дополнительную энергию.

\section{СПИСОК ЛИТЕРАТУРЫ}

1. Тяглов С. Г. Принцип многоуровневого управления устойчивым развитием региональной экономики в части обеспечения эколого-эконо- мических императивов // Пространство экономики. 2014. №2-3. URL: https://cyberleninka.ru/article/n/printsip-mnogourovnevogo-upravleniya-ustoychivym-razvitiem-regionalnoy-ekonomiki-v-chasti-obespecheniya-ekologo-ekonomicheskih

2. Лившиц В. Н. Системный анализ рыночного реформирования нестационарной экономики России: 1992-2013. - М.: ЛЕНАНД, 2013.

3. Дроздов Н. Д., Чернышов В. Н. и др. Системный анализ / Гуманитарная энциклопедия: Концепты [Электронный ресурс] // Центр гуманитарных технологий, 2002-2021. URL: https:/gtmarket.ru/concepts/7111

4. Эшби У. Р. Введение в кибернетику. - М.: КомКнига, 2005.

5. Хомяков $B$. Н. Кибернетика, закон необходимого разнообразия и разработка прогнозов экономических показателей. Часть $1 / /$ Известия ТулГУ. Экономические и юридические науки. Вып. 1. Ч. 1. Тула: Изд-во ТулГУ, 2014. С. 128-141.

6. Число муниципальных образований по субъектам Российской Федерации на 1 января 2020 года // ФСГС.

URL: http://www.gks.ru/free_doc/new_site/bd_munst/1-adm_2020.xlsx

7. Артюхов В., Мартынов А. Закономерности, системные аналоги и теоретические основы мониторинга глобального кризиса, свидетелями и участниками которого мы являемся // Интерфакс-ЭРA. URL: https://openera.ru/storage/files/prognozrazvitiya.pdf 
PRINCIPLES OF THE FORMATION OF THE STRATEGIC MANAGEMENT SYSTEM AT THE LEVEL OF MUNICIPALITIES

\section{Livchits V.N., Myznikova M.N., Dmitrieva O.V.}

Russian municipalities play a key role in the national economy, nevertheless they more and more often face problems that hinder their full development. The main purpose of this article is formulating the basic principles of the formation of the strategic management system at the level of municipalities, which will help improve the quality of life of the population. 